\title{
Validación y adaptación de la escala de control psicológico del profesor hacia las clases de educación física y su efecto sobre las frustraciones de las necesidades psicológicas básicas Validation and adaptation to the Physical Education context of the Psychological Control Teaching Scale, and its effect on the frustration of basic psychological needs
}

\author{
*Rubén Trigueros, *José Manuel Aguilar-Parra, **Josefa González-Santos, *Adolfo Javier Cangas
}

*Universidad de Almería (España), **Universidad de Burgos (España)

Resumen. El papel que adopte el profesor durante las clases de EF es fundamental para conseguir que el alumnado se implique activamente. El objetivo del estudio consistió en validar y adaptar la Escala Docente de Control Psicológico (PCT) al contexto español de EF, con el fin de tener una escala para medir el control psicológico del profesor sobre el alumnado y analizar la relación existente respecto a las cuatro frustraciones de las necesidades psicológicas. Para responder al objetivo del estudio se han empleado dos muestras independientes pertenecientes a varios centros educativos de secundaria. Para analizar las propiedades psicométricas del PCT se ha realizado un análisis factorial confirmatorio, una análisis de invarianza respecto al género y una análisis de fiabilidad. Para analizar la relación entre el control del docente y la frustración de las cuatro necesidades psicológicas se realizó un modelo de ecuaciones estructurales. Los resultados han apoyado la validez y fiabilidad de la estructura del cuestionario, mostrándose invariante respecto al género. El control psicológico ejercido por el docente actuó como predictor de los factores que integran la frustración de las necesidades psicológicas básicas, estando en línea con los postulados de la teoría de la autodeterminación.

Palabras clave: Psicología de la educación, profesores de Educación física, necesidades psicológicas, modelo de ecuaciones estructurales.

\begin{abstract}
The role that teachers adopt during PE classes is fundamental to ensure that students are actively involved. The aim of the study was to validate and adapt the Psychological Control Teaching Scale (PCT) to the Spanish context of PE, in order to have a scale to measure teachers' psychological control over students and to analyze its relationship with the four frustrations of psychological needs. To answer the objective of the study, two independent samples belonging to several secondary schools were selected. To analyze the psychometric properties of the PCT, confirmatory factorial analysis, as well as invariance analysis by gender and reliability analysis were carried out. To analyze the relationship between teachers' control and frustration of the four psychological needs, a model of structural equations was built. The results supported the validity and reliability of the structure of the questionnaire, the model being invariant by gender. Teachers' psychological control acted as a predictor of the factors that trigger frustration of basic psychological needs, our outcomes being in line with the postulates of the theory of self-determination.
\end{abstract}

Keywords: Educational psychology, Physical Education teachers, psychological needs, structural equation models.

\section{Introducción}

Las clases de Educación Física (EF) resultan ser el medio ideal para que el profesorado pueda fomentar hábitos de vida saludables entre los estudiantes de EF, ya que esta asignatura tiene su foco de atención en la implementación de propuestas académicas para el desarrollo de competencias que tiene como finalidad la promoción de hábitos de vida saludables en virtud de la practica regular de actividad física (Vázquez y Márquez, 2017; Zueck, Ramírez, Rodríguez y Irigoyen, 2020). Para ello, se requiere de un papel activo por parte del docente, cuya interacción con el alumnado se antoja indispensable para que este consiga entender los beneficios de la actividad física, tanto a nivel psicológico, conductual como social, y así consolidar hábitos activos y alimenticios saludables (Márquez y Celis, 2016; Tapia, 2019).

Desde la Teoría de la Autodeterminación (TAD; Deci y Ryan, 2008) se sugiere que la actitud que puede adoptar el docente durante sus clases de EF respecto al alumnado puede ser analizada desde dos vertientes, el apoyo a la autonomía o el uso de conductas que buscan el control. El primero de ellos hace referencia al fomento por parte del docente de la iniciativa propia del alumnado, es decir, la capacidad auto decisoria, que se encuentra ligada a la autoiniciación, reco-

Fecha recepción: 14-03-19. Fecha de aceptación: 1-08-19 Rubén Trigueros

rtrigueros@nebrija.es nocimiento de la perspectiva de los alumnos, el interés por el aprendizaje y la mejora de las propias habilidades (Cheon, Reeve, Yu y Jang, 2014; Jang, Reeve y Deci, 2010; Rius y Torrebadella, 2018). Por el contrario, las conductas controladoras del docente, se refieren al uso de comportamientos a menudo sutiles e intrusivos, que buscan presionar a los estudiantes para actuar, pensar y sentir de manera particular (Bartholomew, Ntoumanis y Thøgersen-Ntoumani, 2011). Por ello, resulta evidente que el control actúa de forma opuesta al apoyo a la autonomía, contraponiéndose a la enseñanza adaptativa que esta última promueve, ya que el control manipula, vigila, invalida y restringe las experiencias y la expresión psicológica, conductual y emocional de los estudiantes, inhibiendo el funcionamiento autónomo (Trigueros, Fernández-Campoy, Alías, Aguilar-Parra y Lozano, 2017).

El uso de las estrategias de control por parte del profesor para conseguir que el alumno se comporte de una determinada manera, se puede realizar de dos formas diferentes. Primeramente, a través de un control interno que hace referencia a desencadenar presiones internas en los alumnos apelando a sentimientos de culpa, ansiedad, vergüenza y frustración. La generación de presiones internas ocurre de manera encubierta y sutil, pero no de forma directa (Bartholomew, Ntoumanis y Thøgersen-Ntoumani, 2010). La segunda forma de control es la presión externa, que se realiza de forma abierta y explicita, y que hace referencia a la activación de un sentido de obligación externa al estudiante, como castigos, presiones, recompensas y amenazas (Bartholomew et al, 2011). Ambas tienen el denominador común de que la enseñanza 
controladora se basa en el uso de estrategias de presión, de tal modo que los profesores obligan a los estudiantes a pensar, actuar, percibir y sentir de una determinada manera ignorando el desarrollo de las necesidades psicológicas y frustrando su desarrollo (Trigueros, et al., 2017).

Sin embargo, las diferentes investigaciones que se han desarrollado en el ámbito educativo de la TAD, se han centrado principalmente en la dimensión positiva de la enseñanza. En este sentido, se ha realizado diversos estudios que se han centrado en la percepción de apoyo a la autonomía de los estudiantes de EF y sus enfoques beneficiosos (Goossens, Cardon, Witvrouw, Steyaert y De Clercq, 2016; Nie, Chua, Yeung, Ryan y Chan, 2015). Como resultado, la parte negativa de la enseñanza apenas ha recibido atención de los investigadores, ensombreciendo el hecho que los docentes pueden ser unos de los focos generadores de un entorno desadaptativo (Soenens, Sierens, Vansteenkiste, Dochy y Goossens, 2012).

Según la TAD este entorno desadaptativo generado por el control psicológico del profesor puede afectar de forma negativa a las necesidades psicológicas básicas, definidas como nutrientes esenciales básicos y universales presentes en todos los seres humanos y que son necesarias para el crecimiento, la salud y el bienestar psicológico (Lamoneda y Huertas-Delgado, 2019; Ryan y Deci, 2017). Estos nutrientes son tres: la autonomía, la competencia y la relación con los demás. En lo que se refiere a la autonomía, es que las personas sean los agentes de su propia acción, sin presiones externas. La competencia, se basa en la necesidad experimentar eficacia en la acción. Por último, la relación con los demás hace referencia a la necesidad de relacionarse y preocuparse por los demás, así como a la inversa, el percibir por parte de ellos esa misma necesidad, es decir, sentirse aceptado y relacionarse con los demás (Deci y Ryan, 2012). Sin embargo, varios estudios realizados por González-Cutre, Sicilia, Sierra, Ferriz y Hagger (2016) y Trigueros, Maldonado, Vicente, González-Bernal, Ortiz y González-Santos (2019) justificaron y pusieron de manifiesto la existencia de una cuarta necesidad psicológica básica, que tiene por nombre novedad y que se entiende como necesidad de experimentar algo que antes no se experimentaba o que se desvía de la rutina diaria. Esta necesidad había sido nombrada anteriormente por Deci y Ryan (2000) cuando definieron la motivación intrínseca como el compromiso activo que adquieren las personas con las acciones que encuentran interesantes y novedosas y suponga un desafío optimo. Esta nueva necesidad psicológica se identifica con la exploración y el interés espontaneo, la asimilación y la superación como una forma de desarrollo cognitivo, emocional y social (Ryan y Deci, 2000).

De esta manera, con el fin de solucionar el vacío existente en relación a los instrumentos que mediera el control psicológico ejercido por el docente, Soenens, et al. (2012) crearon la Psychologically Controlling Teaching Scale (PCT) para disponer de una herramienta eficaz con la que medir el estilo de enseñanza controlador del docente, ya que hasta este momento, una baja percepción de apoyo a la autonomía estaba asociada a un uso de técnicas de control. A pesar de ello, tal y como afirman Soenens, et al., (2012), el uso de una puntuación baja de apoyo a la autonomía como predictor de la percepción de un estilo controlador, no resultaría viable ya que, los ítems de la escala de apoyo a la autonomía solo recogen las experiencias psicológicas positivas, de tal manera que resultaría poco probable que se dispongan a los aspectos negativos de estas experiencias. De esta forma, Soenens, et al., (2012) utilizaron una muestra de 533 estudiantes de secundaria con una edad media de 16.9 años para validar la escala. Para ello, realizaron un análisis factorial exploratorio y un análisis de consistencia interna, en ambos análisis los resultados fueron satisfactorios. En este sentido, al análisis factorial exploratorio reveló la existencia de un único factor que representó el 46\% de la varianza, con todas las cargas de los ítems por encima de .58 (valor propio de 3.20). El análisis de consistencia interna a través del alpha de Cronbach fue de .80 .

Paralelamente a este estudio, estos mismos autores realizaron un segundo estudio donde a través de un modelo de ecuaciones estructurales, relacionaron las conductas de control del profesor respecto a la autonomía hacia el estudio, el uso de estrategias meta cognitivas para el aprendizaje y rendimiento académico. Los resultados revelaron que las conductas controladoras generaban en el alumnado una respuesta desadaptativa que actuaban como impedimento para el uso de las estrategias de aprendizaje significativo, afectando al rendimiento académico. De la misma forma, pero en el ámbito del deporte, un estudio realizado por Gurrola, Rodríguez, Walle, Marco, y Zamarripa (2016) mostró como las conductas controladoras del entrenador percibidas por los atletas predecía positivamente la frustración de las necesidades psicológicas básicas y negativamente la motivación autónoma.

Por todo ello, presentamos dos estudios con muestras independientes donde nos proponemos abordar dos objetivos: en el primero (estudio uno), nos proponemos adaptar y validar la PCT al contexto español de la EF demostrando su validez y fiabilidad, realizando para ello los siguiente análisis: un análisis factorial confirmatorio, estadísticos descriptivos y análisis del alfa de Cronbach. Además, con el fin de analizar si el cuestionario es entendido de forma similar por chicos y chicas se realizará un análisis de invarianza respecto al género. En segundo lugar (estudio dos), pretendemos relacionar a través de un modelo de ecuaciones estructurales (MEE) el estilo controlador del docente respecto a la frustración de las necesidades psicológicas básicas. La hipótesis que se plantea es que el estilo controlador del profesor favorecerá la frustración de las necesidades psicológicas.

\section{Estudio 1}

\section{Participantes}

Los participantes en este estudio uno fueron 421 estudiantes de EF (206 chicos y 215 chicas) con edades comprendidas entre los 13 y 19 años $(M=15.28, D T=1.14)$ de dos centros educativos de secundaria de dos provincias del sur de España.

\section{Medidas}

Para medir el control psicológico del profesor se utilizó la Psychologically Controlling Teaching Scale (PCTs; Soenens, et al., 2012). La escala estuvo precedida por el en- 
cabezamiento «Mi profesor de EF...» y consta de 7 ítems con un único factor. Los estudiantes tuvieron que responder según una escala Likert que oscilaba entre 1 (totalmente desacuerdo) a 5 (totalmente de acuerdo).

\section{Procedimiento}

Con el objetivo de poder llevar a cabo la validación del cuestionario al contexto español de EF, se utilizó la estrategia de traducción inversa (Hambleton, 1996). Este proceso consiste en que el cuestionario original fue traducido al español por un grupo de traductores expertos y posteriormente otro grupo lo tradujo a su idioma original. La bondad de la traducción se juzgó en función del grado de coincidencia con la versión original. La versión obtenida fue analizada por tres expertos (Lynn, 1986) en EF, de tal modo que se garantizara que los ítems obtenidos estuvieran bien diseñados para medir el constructo que quería medirse, sin perder el significado original.

Una vez obtenido el cuestionario se contactó con diversos centros educativos de la provincia de Almería, a los que también se les informó del objetivo de la investigación y solicitó su colaboración. A los sujetos se le requirió una autorización paterna o materna para su participación, ya que eran menores de edad. La administración del cuestionario se realizó bajo la supervisión de un encuestador experto miembro del grupo de investigación, que explicó y solventó las dudas que surgieron al cumplimentarlo. El tiempo estimado para completar el cuestionario estuvo en torno a 15 minutos. El estudio recibió la aprobación del comité de ética de la universidad antes de la recopilación de datos.

\section{Análisis de datos}

Con el fin de determinar la validez y fiabilidad de la PCTs en el contexto español de EF se analizó las propiedades psicométricas del cuestionario. En primer lugar, se realizó un análisis factorial confirmatorio (AFC) para testar la estructura factorial. En segundo lugar, se llevó a cabo análisis multigrupo para analizar la invarianza respecto al género El criterio mínimo para aceptar que la estructura del cuestionario se muestra invariante respecto al género, es la ausencia de diferencias significativas entre el modelo 1 y el modelo 2 (Marsh, 1993). A continuación, se realizó análisis estadísticos descriptivos y se testó la fiabilidad del instrumento a través de análisis de consistencia interna (alfa de Cronbach). Para los análisis de datos se utilizaron los paquetes estadísticos SPSS 19.0 y AMOS 19.0.

Debido a que el coeficiente de Mardia resultó ser alto $(82,34)$ para el AFC se utilizó el método de estimación de máxima verosimilitud junto con el procedimiento de bootstrapping. Los estimadores no resultaron afectados por la falta de normalidad, por lo que se considerados robustos (Byrne, 2001). Con el objetivo de aceptar o rechazar el modelo testado, se tuvo en consideración un conjunto de índices de ajuste: $\div$ 2/gl, CFI (Comparative Fit Index), IFI (Incremental Fit Index), RMSEA (Root Mean Square Error of Approximation) más su intervalo de confianza (IC) al 90\%, y SRMR (Standardized Root Mean Square Residual). Dado que el $\div 2$ es muy sensible al tamaño muestral (Jöreskog y Sörbom, 1993), se empleó el $\div$ 2/gl, considerándose aceptables valores inferiores a 5 (Bentler, 1989). Los índices incrementales (CFI e IFI) muestran un buen ajuste con valores iguales o superiores .95 (Schumacker y Lomax, 1996), mientras que los índices de error (RMSEA y SRMR) se consideran aceptables con valores iguales o menores de .08 (Browne y Cudeck, 1993; Hu y Bentler, 1999).

\section{Resultados}

\section{Análisis factorial confirmatorio}

Los índices de ajuste del modelo testado (Figura 1) fueron apropiados: $x 2(14 . \mathrm{N}=421)=45.99, p<.01 ; x 2 / g l=3.29$; $\mathrm{CFI}=.98$; IFI = .98; RMSEA = .074 (IC 90\% = .051-.098); SRMR $=.026$. Los pesos de regresión estandarizados se encontraban entre.75 y .84, siendo estadísticamente significativos $(p<.001)$.

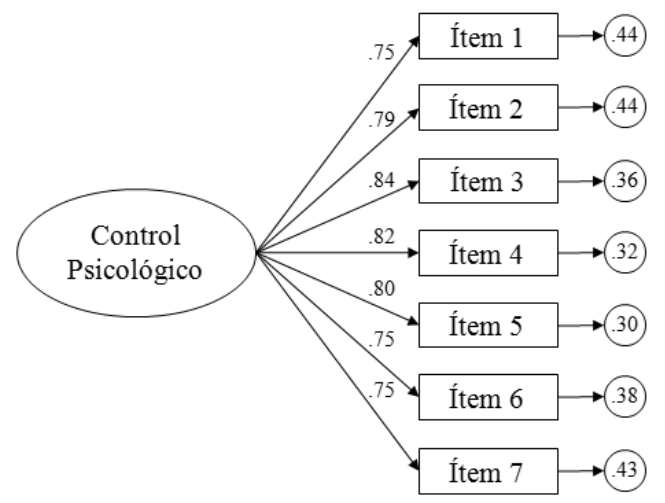

Figura 1. Análisis factorial confirmatorio del PCT. Las elipses representan los factores y los rectángulos representan los diferentes ítems. Las varianzas residuales se muestran en los círculos pequeños.

\section{Análisis de invarianza por sexo}

Para conocer si la estructura factorial de la escala se muestra invariante respecto al género, se realizó un análisis multigrupo. Tal y como muestra la tabla 1 , no se observaron diferencias significativas en el estadístico $\div 2$ entre el modelo 1 (modelo sin restricciones) y el modelo 2 (modelo de invarianza en los pesos de medida). Por otra parte, los resultados sí mostraron diferencias significativas entre el modelo $1 \mathrm{y}$ el modelo 3 (modelo de covarianzas estructurales invariantes) y el modelo 4 (modelo de residuos de medida invariantes).

\section{Estadísticos descriptivos y de fiabilidad}

Según se observa en la tabla 2, el alumnado participante en este estudio obtuvo una puntuación media para la percepción del control psicológico del profesor de 2.73. Además, se realizó un análisis de consistencia interna (ver Tabla 2) revelando un valor de alfa de Cronbach de .92 para estilo controlador del profesor.

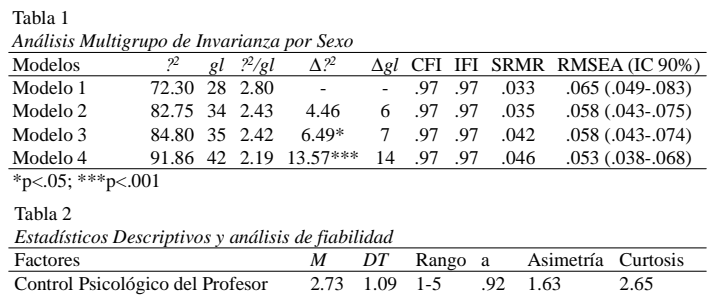




\section{Estudio 2}

\section{Participantes}

En este estudio dos participó una muestra independiente de 428 estudiantes de EF diferente al estudio anterior, donde 211 eran chicos y 217 eran chicas con edades comprendidas entre los 13 y 19 años ( $(M=15.56, \mathrm{DT}=1.08)$ de dos centroseducativos de secundaria de dos provincias del sur de España.

Estilo controlador. Con el fin de medir el control del docente percibido por los estudiantes en el contexto de las clases de EF, se utilizó la versión adaptada y validada en el estudio anterior. La escala está precedida por el siguiente encabezamiento «Mi profesor de EF...» y consta de 7 ítems (e.g., «Me hace sentir culpable cuando no logro cumplir sus expectativas») con un único factor. Los estudiantes debían de responder según una escala Likert que oscilaba entre 1 (totalmente desacuerdo) a 5 (totalmente de acuerdo).

Frustración de las necesidades psicológicas. Se empleó la versión de Escala de la Frustración de las Necesidades Psicológicas hacia las clases de EF de Trigueros et al (2019). La escala está precedida por el encabezamiento «En mis clases de EF...» y consta de 17 ítems, repartidos entre cada uno de los factores que componen la escala autonomía (e.g., «Me siento presionado/a a comportarme de una cierta manera»), competencia (e.g., «Hay actividades que me hacen sentir incapaz»), relación con los demás (e.g., «Me siento rechazado/a por mis compañeros/as de clase») y novedad (e.g., «Siento frecuentemente cierta monotonía»). Los estudiantes tuvieron que responder según una escala Likert que oscilaba entre 1 (nada verdadero) a 7 (totalmente verdadero).

\section{Procedimiento}

Para poder llevar a cabo este segundo estudio, se solicitó en primer lugar el consentimiento informado a los familiares del alumnado para que pudieran participar en el estudio, ya que eran menores de edad. En segundo lugar, se les informó que participarían en una encuesta sobre su motivación en educación física. Los cuestionarios se completaron anónimamente en el aula bajo la supervisión del investigador. El estudio recibió la aprobación del comité de ética de la universidad antes de la recopilación de datos.

\section{Análisis de datos}

En este segundo estudio, se realizó un análisis de estadísticos descriptivos, correlaciones bivariadas y análisis de fiabilidad utilizando el programa estadístico SPSS versión 19. Además, se realizó un AFC del modelo y MEE utilizando para ello el programa AMOS versión 19 (Jöreskog y Sörbom, 2003) con el fin de testar las relaciones establecidas en el modelo hipotetizado.

Con el objetivo de aceptar o rechazar el modelo testado, se tuvo en consideración los índices de ajuste establecidos en el estudio 1. Utilizando para el AFC y el MEE el método de estimación de máxima verosimilitud junto con el procedimiento de bootstrapping debido a que el coeficiente de Mardia fue alto $(53,12)$. Para realizar los análisis de validez predictiva a través del MEE se realizó el método de dos pasos propuesto por Anderson y Gerbing (1988). Este método consiste en la realización de AFC de cada una de las escalas o cuestionarios que se han usado en este estudio. Posteriormente, el paso es testar el MEE y analizar las relaciones existente entre cada una de las variables usadas en el modelo. Para poder realizarlo, en cada factor se reducen el número de variables latentes, este paso resulta idóneo cuando el tamaño muestral resulta ser no demasiado grande, en comparación al número de variables latentes (Marsh, Richards, Johnson, Roche, y Tremayne, 1994; Vallerand, 2007). El criterio para que la reducción sea lograda es mediante la combinación de pares ser más fiable, ya que tienden a ser distribuidos de forma normal, además de reducir a la mitad la ratio del número de variables medidas en el modelo. De esta forma, la primera mitad de los ítems de cada subescala conforma el primer bloque, mientras que la segunda mitad de ítems conforman el segundo bloque.

\section{Resultados}

\section{Análisis Preliminar}

Los estadísticos descriptivos y correlaciones bivariadas a través del á de Cronbach entre las variables del estudio se puede observar en la tabla 3. En ella, las puntuaciones medias de los factores estuvieron por debajo del punto intermedio, destacando 1.69 para control del profesor como la puntuación media inferior y en el extremo opuesto se encuentra la puntuación de 2.18 para frustración de la relación con los demás.

En cuanto a los análisis de correlación reflejó una asociación positiva entre cada uno de los factores que integran este estudio, mostrando una significatividad de $p<.01 \mathrm{y}$ $p<.05$.

\begin{tabular}{|c|c|c|c|c|c|c|c|c|}
\hline Factores & $M$ & $D T$ & Rango & $\mathrm{a}$ & 2 & 3 & 4 & 5 \\
\hline 1. Control Psicológico del Profesor & 1.69 & .81 & $1-5$ & .82 & $.87 * *$ & $.56^{* *}$ & $18^{* *}$ & $.29 * *$ \\
\hline 2. Frustración Autonomía & 1.95 & 1.11 & $1-7$ & .84 & & $.56 * *$ & $16^{* * *}$ & $.29 * *$ \\
\hline 3. Frustración Novedad & 2.08 & 1.04 & $1-7$ & .80 & & & $.11 *$ & $.19 * *$ \\
\hline 4. Frustración Relación con los demás & 2.18 & 1.45 & $1-7$ & .89 & & & & $.15^{* *}$ \\
\hline 5. Frustración Competencia & 2.09 & 1.36 & $1-7$ & .85 & & & & \\
\hline
\end{tabular}

\section{Análisis Factoriales Confirmatorios y Modelo de Ecuaciones Estructurales}

Los índices de ajuste de los AFC de cada una de las escalas han mostrado unos índices de ajuste adecuados siendo de: $x 2(14 . \mathrm{N}=428)=37.183, \mathrm{p}<.01 ; x 2 / g l=2.66 ; \mathrm{CFI}=.99$; IFI $=.99 ;$ RMSEA $=.062($ IC $90 \%=.037-.088) ;$ SRMR $=.022$ para control percibido y de $\div 2(113 . \mathrm{N}=428)=214.949, p<$ $.001 ; \mathrm{x} 2 / \mathrm{gl}=1.90 ; \mathrm{CFI}=.98 ; \mathrm{IFI}=.98 ; \mathrm{RMSEA}=.046$ (IC $90 \%$ $=.037-.055) ; \mathrm{SRMR}=.046$ para frustración de las necesidades psicológicas básicas.

El modelo de relaciones predictivas hipotetizado (Figura 2), ha mostrado que los índices de ajuste fueron adecuados: $(31, \mathrm{~N}=428)=66.119, x 2 / g \mathrm{l}=2.13, p<.001, \mathrm{IFI}=.99, \mathrm{CFI}=.99$, RMSEA = .052. $($ IC $90 \%=.034-.069)$, SRMR $=.035$. Estos resultados se ajustan a los parámetros establecidos, por lo que podemos aceptar como adecuado el modelo propuesto (Hu y Bentler, 1999). De igual modo, la contribución de cada uno de los factores a la predicción de otras variables se examinó a través de los pesos de regresión estandarizados. En este sentido, en la figura 2, se puede observar como el control ejercido por el profesor predijo positivamente la frus- 


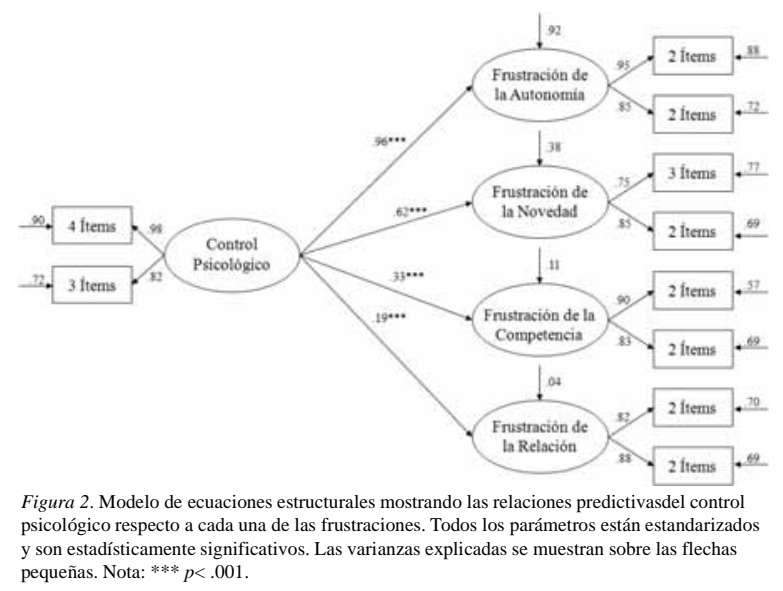

tración de la autonomía $(\beta=.96, \mathrm{p}<.001)$, la competencia $(\beta=$ .33 , $\mathrm{p}<.001)$, la relación con los demás $(\beta=.19$, $\mathrm{p}<.001)$ y la novedad $(\beta=.62, \mathrm{p}<.001)$.

\section{Discusión}

A través del presente estudio hemos tratado de mostrar evidencias del papel que puede tener el docente desde la perspectiva de control psicológico, que implica una comunicación de actitud de aprobación condicional y practicas intrusivas y manipuladoras sobre el malestar psicológico de los estudiantes en el contexto de las clases de EF. Concretamente, examinamos la asociación entre el control percibido sobre la frustración de las necesidades psicológicas básicas. Sin embargo, previamente analizamos la validez y la fiabilidad del PCT con el fin de adaptarlo y validarlo al contexto español de EF y tener una herramienta eficaz con la cual evaluar el control ejercido por el profesor desde la percepción de los alumnos.

En este sentido, los resultados del AFC del PCT mostraron unos índices de ajuste aceptables. Del mismo modo, los análisis de fiabilidad obtuvieron unos índices de ajuste igualmente aceptables con una puntuación superior a .80 en el valor de consistencia interna a través del alfa de Cronbach. Además, el análisis de invarianza respecto al sexo se mostró invariante, por lo que futuros estudios podrán realizar estudios comparativos entre chicos y chicas respecto al control percibido en EF ya que los ítems del cuestionario son entendidos de forma similar por las dos poblaciones.

Esto resultados se muestran en línea al cuestionario original desarrollado por (Soenens et al., 2012). En este sentido, el cuestionario original obtuvo una puntuación por encima de .80 en el alpha de Cronbach y unos índices de ajuste aceptables para el AFC. Sin embargo, los autores del cuestionario original no realizaron un análisis de invarianza, por lo que no podemos comparar los resultados del presente estudio.

En cuanto a los índices de ajuste del MEE usado para analizar la validez predictiva del control percibido respecto a la frustración de las necesidades psicológicas, reveló unos índices de ajuste aceptables mostrando que el control percibido se relaciona de forma positiva con la frustración de competencia, de novedad, de autonomía y de relación con los demás.

Sin embargo, estos resultados apenas pueden compa- rarse con estudios previos en el ámbito de las clases de EF debido a que las diferentes investigaciones que se han desarrollado en el ámbito educativo de la TAD se han centrado principalmente en la dimensión positiva de la enseñanza. A pesar de ello, recientemente han surgido diferentes estudios que relacionan el ejercicio controlador del docente durante las clases de EF sobre el bienestar psicológico del alumnado (Bartholomew, et al., 2018; Haerens, Aelterman, Vansteenkiste, Soenens y Van Petegem, 2015) y del entrenador sobre las necesidades psicológicas y la motivación (Langan, Toner, Blake y Lonsdale, 2015).

En este sentido, el estudio realizado por Soenens, et al., (2012) mostró como el uso de técnicas basadas en el control del docente se relacionaba de forma negativa con el aprendizaje profundo, y positivo con el malestar psicológico definido por la despersonalización del alumnado, teniendo como consecuencias la inhibición de conductas positivas como el tiempo de estudio. Del mismo modo, un estudio realizado por Bartholomew, et al., (2018), destaca el efecto positivo que posee el control docente sobre la frustración de las necesidades psicológicas, y negativo sobre la motivación autónoma y la autoestima. En el mismo sentido, pero en el ámbito deportivo, el estudio realizado por Trigueros (2017) analizó el efecto del uso de técnicas controladoras por parte del entrenador sobre las necesidades psicológicas, mostrando una asociación positiva respecto a la frustración de las necesidades psicológicas y negativa respecto a la satisfacción de las necesidades psicológicas y teniendo como consecuencia una limitación en el desarrollo de la motivación autónoma y en el desarrollo del bienestar psicológico representada como la resiliencia. Sin embargo, los estudios de Bartholomew, et al., (2018) y Trigueros (2017) analizaron el efecto de las conductas controladoras sobre la frustración de las necesidades psicológicas pero desde un punto de vista general, es decir, agrupando las necesidades psicológicas de competencia, autonomía y relación con los demás en un único factor. La novedad no se incluyó en esos estudios debido a que es un constructo de reciente incorporación, realizado por Trigueros et al (2019).

En general, estos datos siguen los postulados de la TAD donde desde la vertiente contraria denominada apoyo a la autonomía,que es aquella donde el docente crea cierta libertad de ejecución para que el alumnado durante las clases de EF se crean las condiciones para que experimenten un sentido de voluntad y selección propia, y que se encuentra ligada a la autoiniciación, al interés por el aprendizaje, la motivación autónoma y la mejora de las propias habilidades (Moreno, et al., 2013).

\section{Limitaciones y prospectivas}

A pesar de que los resultados del presente estudio muestran un apoyo psicométrico para el PCT, es necesario mostrar algunas de las limitaciones que posee, En primer lugar, resulta necesario que futuros trabajos exploren y analicen la estructura factorial de la escala en otros estudiantes con diferentes características (eg. edad, curso, estatus socioeconómico, etc.) ya que la validación de un instrumento ha de ser un proceso continuo. Seguidamente, con el fin de mostrar evidencias de la validez predictiva de la escala, 
futuros trabajos deberían considerar variables que actúen como precedentes y/o consecuentes del control psicológico percibido.

Por otro lado, en relación al modelo predictivo, resulta necesario recalcar que se trata de un estudio relacional por lo que no permite extrapolar relaciones de causa-efecto y que los resultados obtenidos podrían ser interpretados de múltiples formas diferentes. Por ello, se ha tratado de exponer posibilidades y no casualidad, con el fin de poder explicar las relaciones existentes entre las variables de ambos estudios.

A pesar de los resultados alcanzados, futuros estudios deberían de profundizar en el efecto del control del docente sobre las diferentes necesidades psicológicas básicas (satisfacción y frustración) además de analizar y discutir los resultados según el nivel educativo y teniendo en cuenta los diferentes contextos educativos, así como otros ámbitos (eg., deportivo o actividad física). Igualmente, futuros estudios deberían tener en cuenta no solo los efectos negativos sino también los positivos y analizar como ambas valencias afecta a la motivación y adopción de hábitos fuera del ámbito escolar de los estudiantes.

\section{Conclusiones}

El presente estudio ha mostrado evidencias de validez y fiabilidad de la PCT en el contexto español de las clases de EF, además de observar el efecto positivo del uso de técnicas controladoras del docente sobre las cuatro frustraciones de las necesidades psicológicas básicas.

\section{Referencias}

Anderson, J. C. y Gerbing, D. W. (1988). Structural equation modeling in practice: A review and recommended twostep approach. Psychological Bulletin, 103(3), 411-423.

Bartholomew, K. J., Ntoumanis, N., Mouratidis, A., Katartzi, E., Thøgersen-Ntoumani, C., y Vlachopoulos, S. (2018). Beware of your teaching style: A school-year long investigation of controlling teaching and student motivational experiences. Learning and Instruction, 53, 50-63.

Bartholomew, K.J., Ntoumanis, N., y Thøgersen-Ntoumani, C. (2010). The controlling interpersonal style in a coaching context: Development and initial validation of a psychometric scale. Journal of Sport and Exercise Psychology, 31, 193-216.

Bartholomew, K., Ntoumanis, N., y Thøgersen-Ntoumani, C. (2011). Self-Determination theory and the darker side of athletic experience: The role of interpersonal control and need thwarting. Sport and Exercise Psychology Review, 7, 23-27.

Bentler, P.M. (1989). EQS structural equations program manual. Los Angeles: BMDP Statistical Software

Browne, M.W., y Cudeck, R. (1993). Alternative ways of assessing model fit. Sage Focus Editions, 154, 136-136.

Byrne, B.M. (2001). Multivariate applications book series. Structural equation modeling with AMOS: Basic concepts, applications, and programming. Mahwah, NJ: Lawrence Erlbaum Associates, 10.

Cheon, S.H., Reeve, J., Yu, T.H., y Jang, H.R. (2014). The teacher benefits from giving autonomy support during physical education instruction. Journal of Sport and Exercise Psychology, 36(4), 331-346.

Deci, E. L., y Ryan, R. M. (2000). The» what» and» why» of goal pursuits: Human needs and the self-determination of behavior. Psychological Inquiry, 11(4), 227-268.

Deci, E. L., y Ryan, R. M. (2008). Self-determination theory: A macrotheory of human motivation, development, and health. Canadian Psychology, 49(3), 182-185.

Deci, E. L., y Ryan, R. M. (2012). Motivation, personality, and development within embedded social contexts: An overview of self-determination theory. The Oxford Handbook of Human Motivation, 85-107.

González-Cutre, D., Sicilia, Á., Sierra,A. C., Ferriz, R., y Hagger, M. S. (2016). Understanding the need for novelty from the perspective of self-determination theory. Personality and Individual Differences, 102, 159-169.

Goossens, L., Cardon, G., Witvrouw, E., Steyaert, A., y De Clercq, D. (2016). A multifactorial injury prevention intervention reduces injury incidence in Physical Education Teacher Education students. European Journal of Sport Science, 16(3), 365-373.

Gurrola, H.B.A., Rodríguez, J.L.T., Walle, J.M.L., Marco, M.I.T., y Zamarripa, J. (2016). Estilos interpersonales del entrenador, frustración de las necesidades psicológicas básicas y el burnout: un análisis longitudinal en futbolistas. Retos: Nuevas Tendencias en Educación Física, Deporte y Recreación, (30), 132-137.

Hambleton, R. K. (1996). Adaptación de tests para su uso en diferentes idiomas y culturas: fuentes de error, posibles soluciones y directrices prácticas. En J. Muñiz (Ed.), Psicometría (pp. 207-238). Madrid: Universitas.

Haerens, L., Aelterman, N., Vansteenkiste, M., Soenens, B., y Van Petegem, S. (2015). Do perceived autonomysupportive and controlling teaching relate to physical education students' motivational experiences through unique pathways? Distinguishing between the bright and dark side of motivation. Psychology of Sport and Exercise, 16, 26-36.

Hu, L.T., y Bentler, P.M. (1999). Cutoff criteria for fit indexes in covariance structure analysis: Conventional criteria versus new alternatives. Structural Equation Modeling: a Multidisciplinary Journal, 6(1), 1-55.

Jang, H., Reeve, J., y Deci, E. L. (2010). Engaging students in learning activities: It is not autonomy support or structure but autonomy support and structure. Journal of Educational Psychology, 102(3), 588-600.

Jöreskog, K. G., y Sörbom, D. (1993). LISREL 8: Structural equation modeling with the SIMPLIS command language. Chicago: Scientific Software.

Jöreskog, K. G., y Sörbom, D. (2003). LISREL 8.54.Structural equation modeling with the Simplis command language. Chicago: Scientific Software.

Langan, E., Toner, J., Blake, C., y Lonsdale, C. (2015). Testing the effects of a self-determination theory-based intervention with youth gaelic football coaches on athlete motivation and burnout. The Sport Psychologist, 29(4), 293-301.

Lamoneda, J. y Huertas-Delgado, F. J. (2019). Basic psychological needs, sport organization and levels of 
physical activity in scholars. Revista de Psicología del Deporte, 28(1), 115-124.

Lynn, M. R. (1986). Determination and quantification of content validity. Nursing Research, 35(6), 382-386.

Márquez, J.M.C., y Celis, C.C. (2016). Hábitos y estilos de vida saludables en la edad escolar. Sevilla: Wanceulen.

Marsh, H.W., Richards, G.E., Johnson, S., Roche, L., y Tremayne, P. (1994). Physical Self-Description Questionnaire: Psychometric properties and a miiltitraitmeltimethod analysis of relations to existing instruments. Journal of Sport and Exercise Psychology, 16(3), 270305.

Nie, Y., Chua, B.L., Yeung, A.S., Ryan, R.M., y Chan, W.Y. (2015). The importance of autonomy support and the mediating role of work motivation for well being: Testing self determination theory in a Chinese work organisation. International Journal of Psychology, 50(4), 245-255.

Rius, J. y Torrebadella, X. (2018). Reflexiones para (re)formular una educación física crítica. Revista Internacional de Medicina y Ciencias de la Actividad Física y el Deporte, 18(71), 441-462.

Ryan, R.M., y Deci, E.L. (2000). The darker and brighter sides of human existence: Basic psychological needs as unifying concept. Psychology Inquiry, 11, 319-338.

Ryan, R. M., y Deci, E. L. (2017). Self-determination theory: Basic psychological needs in motivation, development, and wellness. Guilford Publications.

Schumacker, R. y Lomax, R.G. (1996) A Beginner ìs Guide to Structural Equation Modelling. Lawrence Erlbaum Associates Inc., Mahwah-NJ.

Sicilia, A., Ferriz, R. y Sáenz-Álvarez, P. (2013). Validación española de la escala de frustración de las necesidades psicológicas (EFNP) en el ejercicio físico. Psychology, Society, \& Education, 5(1), 1-19.

Soenens, B., Sierens, E., Vansteenkiste, M., Dochy, F., y Goossens, L. (2012). Psychologically controlling teaching: Examining outcomes, antecedents, and mediators. Journal of Educational Psychology, 104(1), 108-120.

Tapia, A. (2019). Diferencias en los niveles de actividad física, grado de adherencia a la dieta mediterránea y autoconcepto físico en adolescentes en función del sexo. Retos: Nuevas tendencias en Educación Física, Deporte y Recreación, 36, 185-192.

Trigueros, R. (2017). La influencia del entrenador sobre la motivación y la resiliencia. Un estudio desde la perspectiva de la teoría de la autodeterminación. Tesis doctoral: Universidad de Almería.

Trigueros, R., Maldonado, J.J., Vicente, F., González-Bernal, J.J., Ortiz, L., y González-Santos, J., (2019). Adaptación y Validación al contexto de la Educación Física de la Escala de la Frustración de las Necesidades Psicológicas (EFNP) en el ejercicio físico, con la inclusión de la novedad como necesidad psicológica. Revista de Psicología del Deporte.

Trigueros, R., Fernández-Campoy, J.M., Alías, A., AguilarParra, J.M. y Lozano M.C. (2017). Adaptación y validación Española del Controlling Coach BehaviorsScale (CCBS). Internacional Journal of Developmental and Educational Psychology, 2(1), 417-428.

Ulstad, S. O., Halvari, H., Sørebø, Ø., y Deci, E. L. (2016). Motivation, Learning Strategies, and Performance in Physical Education at Secondary School. Advances in Physical Education, 6(01), 27-41.

Vallerand, R. J. (2007). A hierarchical model of intrinsic and extrinsic motivation for sport and physical activity. In M. S. Hagger \& N. L. D. Chatzisarantis (Eds.), Intrinsic motivation and self-determination in exercise and sport (pp. 255-279,356-363). Champaign, IL: Human Kinetics.

Vera-Estrada, F., Sánchez-Rivas, E. y Sánchez-Rodríguez, J. (2018). Promoción de la actividad física saludable en el recreo escolar. Revista Internacional de Medicina y Ciencias de la Actividad Física y el Deporte,18(72), 655-668.

Vázquez, P. G., y Márquez, A. R. (2017). La actividad física como fuente de salud y calidad de vida. Sevilla: Wanceulen.

Zueck, M. C., Ramírez, A. A., Rodríguez, J. M. y Irigoyen, H. E. (2020). Satisfacción en las clases de Educación Física y la intencionalidad de ser activo en niños del nivel de primaria. Retos: Nuevas tendencias en Educación Física, Deporte y Recreación, 37, 33-40.

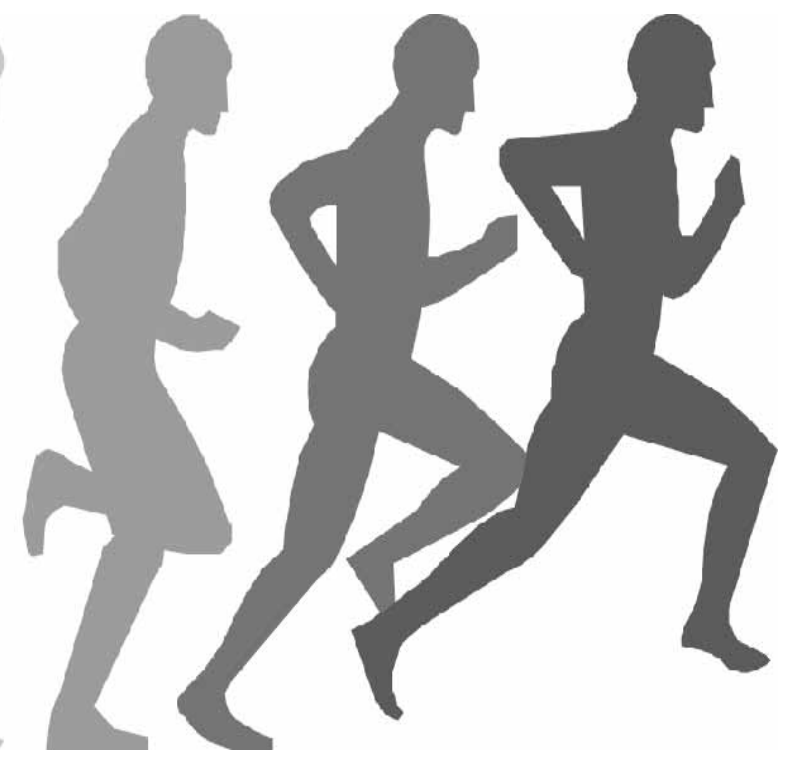

